\title{
Diagnosis and excision of an ulnar artery aneurysm in a two-year-old boy
}

\author{
Ibrahim Amjad MD, Travis Murphy BS ${ }^{1}$, Evan Zahn MD²
}

I Amjad, T Murphy, E Zahn. Diagnosis and excision of an ulnar artery aneurysm in a two-year-old boy. Can J Plast Surg 2010;18(1):e15-e16.

Ulnar artery aneurysms in children younger than 10 years of age are rare, with fewer than 10 reported cases worldwide. Unlike adult arterial aneurysms, the etiology of these lesions in children is not well understood, and there is no accepted method for treating these lesions. The method of diagnosis and excision of an ulnar artery aneurysm that showed a favourable outcome in a two-year-old boy is presented. Different approaches to treating similar lesions have been reported, and these approaches are briefly reviewed.

Key Words: Children; Pediatric; Traumatic aneurysm; Ulnar artery aneurysm

\section{Diagnostic et exérèse d'un anévrysme de l'artère cubitale chez un garçonnet de deux ans}

Les anévrysmes de l'artère cubitale chez les enfants de moins de 10 ans sont un phénomène rare, puisqu'on en dénombre moins de 10 cas dans le monde. Contrairement aux anévrysmes artériels chez l'adulte, l'étiologie de ces lésions chez les enfants est mal élucidée et il n'y a pas de méthode thérapeutique clairement établie pour les corriger. Les auteurs présentent ici la méthode diagnostique et thérapeutique utilisée avec succès chez un garçonnet de deux ans, et donnent un aperçu de quelques autres approches qui ont été ont été décrites pour ce genre de lésion.

U

Inar artery aneurysms are exceedingly rare in children younger than 10 years of age, with fewer than 10 cases reported worldwide. Secondary to their rarity, the etiology of these lesions is not yet understood. Ulnar artery aneurysms have been characterized in the adult population, usually occurring secondary to occupationally acquired, repetitive stress disorders such as hypothenar hammer syndrome (1-3). The present case report describes the course of treatment for an ulnar aneurysm in the wrist of a two-year-old boy, along with a brief review of the existing literature detailing other approaches to management.

\section{CASE PRESENTATION}

A two-year-old boy presented with a bump on the left arm, which was noticed by the mother during a trip to the emergency room for suspected trauma to the wrist. The mass was originally believed to be a ganglion cyst. It was not known whether the mass had changed in size between the time of discovery and the time of visit to the office. Apart from the visit to the emergency room, the patient's medical history involved a case of omphalitis at 21 days and a frenectomy for tongue-tie at 1.5 months of age, with no history of arterial line placement. The patient's family history of similar lesions was negative.

Ultrasound characterized the mass as an ovoid lesion, with the possibility of being a large pseudoaneurysm of the ulnar artery.

On physical examination in the office, the mass on the left wrist appeared to be a ganglion cyst. Neither bruit nor thrill were noted, and neurovascularity was normal. No finger ischemia was noted.

Magnetic resonance angiography identified the mass on the volar aspect of the left wrist to be a large pseudoaneurysm of the distal ulnar artery just anterior to the wrist. The dimensions were approximately $1.8 \mathrm{~cm}$ in length by $1.0 \mathrm{~cm}$ in width by $1.2 \mathrm{~cm}$ in height. Heterogeneous signal intensities with hyperdense elements on T1 were suggestive of thrombus. No arteriovenous malformations were noted. Magnetic resonance imaging suggested a true aneurysm with thrombosis. The lesion was not

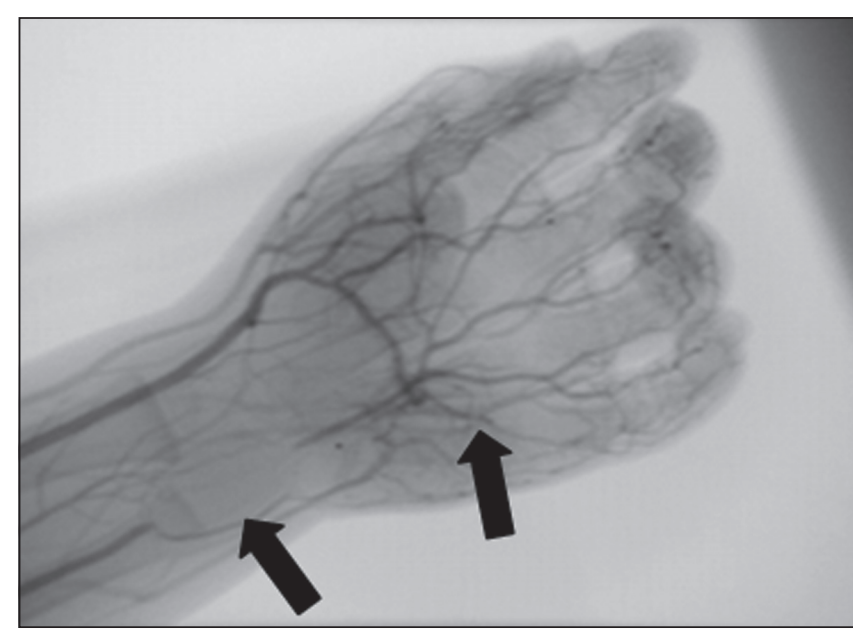

Figure 1) Fluoroscopic angiography of the ulnar and radial arteries showing an occluded ulnar artery at the point of aneurysm and complete perfusion of digits via the radial system and palmar arch (arrows)

determined to occlude the ulnar artery, and an angiographic arteriogram was to be performed to determine both the characteristics of the lesion and whether repair of the ulnar artery was necessary after excision of the aneurysm. Consultation with a geneticist was also elected to investigate possible clinically important congenital or connective tissue disorders such as Kawasaki, Marfan's or Ehlers-Danlos syndromes because they have been implicated in the development of ulnar artery aneurysms $(4,5)$. Based on meeting with the geneticist, the patient was determined to have no connective tissue disorders.

Intraoperative arteriogram, 16 days after the magnetic resonance imaging/magnetic resonance angiography, noted that the ulnar artery was completely occluded (Figure 1). No contrast entered the lesion itself and no emboli were noted. A long gap between the end of contrast and the level of the wrist was

${ }^{1}$ Florida International University; ${ }^{2}$ Congenital Heart Institute, Miami Children's Hospital, Miami, Florida, USA

Correspondence and reprints: Dr Ibrahim Amjad, 1100 South West 57th Avenue, West Miami, Florida 33144, USA.

Telephone 305-267-8885, fax 305-265-0142, e-mail doctor@driamjad.com 
noticed, representing the aneurysm itself. Good filling of the palmar arch and all digits of the left hand via the radial artery system was noted (Figure 1). Viability of complete excision of the lesion without repair of the ulnar artery was confirmed, and resection of the thrombosed aneurysm was performed.

In the operating room, the area over the mass was incised and dissected. Proximal control of the ulnar artery was gained with a right-angle clamp. The medial nerve and tendons were noted and were uncompromised. On full dissection, clips were applied to each end of the lesion with a small clip applier and tied with 5-0 silk. Once the clips had been placed and tied, the lesion was fully removed and the area was copiously irrigated. No bleeding from either end of the severed ulnar artery was noted. A volar splint was placed to stabilize the wrist and to prevent the patient from disrupting the stitches.

The mass excised from the arm was sent to surgical pathology for analysis. The mass was determined to be an 'arterial aneurysm, left ulnar' and diagnosed as a 'thrombosed blood vessel with organization'.

The patient recovered well from the surgery and was discharged the same day. Twelve days postoperatively, his wrist was determined to be doing well and the splint was removed. Forty days postoperatively, the patient had good neurovascular status without problems.

\section{DISCUSSION}

In the pediatric population, the extreme rarity of these aneurysms is largely responsible for the lack of deep understanding of their etiology (1-3). A search of electronic databases resulted in fewer than 10 previously reported cases of ulnar artery aneurysm in children younger than 10 years of age worldwide. However, ulnar artery aneurysms are relatively well understood in adults, usually arising from repetitive stress on the arterial wall (1-3). The following general characteristics of the different types of aneurysms are based on a wide range of patient age groups.

True aneurysms are thought to arise from processes associated with Kawasaki, Marfan's or Ehlers-Danlos syndromes that damage or weaken the arterial wall (4).

Pseudoaneurysms are suspected to arise from traumatic or extravascular events that weaken or damage the arterial wall $(4,6)$. These events can range from venipucture to tumoral growth that interferes with the integrity of the arterial wall (6). Trauma to areas in which the artery is particularly close to the surface is commonly attributed to arterial pseudoaneurysms (6).

Among the reported cases, some involved ulnar artery aneurysm in conjunction with known trauma, while others reported aneurysms with possible connective tissue or congenital factors that could have led to the development of the lesion. In particular, Kawasaki and Marfan's syndromes have been implicated in ulnar artery aneurysm cases, but not in children younger than 10 years of age $(4,5)$.

General methods for diagnosing these lesions include nonand fairly invasive procedures and should be suited to the patient, severity of the lesion and skills of the diagnostic staff. Using an Allen's test by hand to determine the presence of the palmar arch has been reported (3), but the accuracy of such a test in infants is questionable. However, performance of an Allen's test using angiography and fluoroscopy can show defects very clearly (3). Ultrasound and magnetic resonance imaging have also been cited as methods for diagnosing ulnar artery aneurysm (3). Ultrasound in particular is advocated by Al-Omran (3) due to its noninvasive nature, ability to identify thrombus and capabilities in determining hand perfusion preand perioperatively. Multidetector row computed tomography angiography has been advocated due to its smaller morbidity rate when compared with conventional catheter arteriography, but this method may not yet be suitable on the smaller scale of pediatrics because artifact formation was problematic in the adult patient, as cited by the authors (7).

All cases of ulnar aneurysm reported in children younger than 10 years of age were eventually corrected surgically, although nonsurgical approaches to treatment have been reported (8). The primary difference in the surgical treatments reported was the choice to reconstruct the area of aneurysm. The decision of whether to reconstruct was universally based on the adequacy of hand perfusion during trial clamping of the ulnar artery. Poor hand perfusion without the ulnar artery system necessitated reconstruction. Reconstruction regardless of hand perfusion without the ulnar artery has been suggested as a method to mitigate potential complications in a hand dependent on the radial artery system $(3,4)$. Different methods of reconstruction have been reported, but none have been shown to be more or less favourable. Among these methods to preserve hand perfusion, reconstruction without severing the ulnar artery and addition of a tubular polytetrafluoroethylene or Dacron envelope has been reported, but such an envelope could prove to be problematic with patient growth (6). Reverse saphenous vein interposition graft (9), ipsilateral dorsal hand vein interposition graft (10) and end-to-end anastomosis of the severed artery have also been reported $(3,11)$.

ACKNOWLEDGEMENTS: The authors acknowledge the support of both the Miami Children's Hospital (USA) interventional cardiology and surgical staff for their assistance in the successful completion of the procedure.

\section{REFERENCES}

1. Ferris BL, Taylor LM Jr, Oyama K, et al. Hypothenar hammer syndrome: Proposed etiology. J Vasc Surg 2000;31:104-13.

2. Cooke RA. Hypothenar hammer syndrome: A discrete syndrome to be distinguished from hand-arm vibration syndrome. Occup Med (Lond) 2003;53:320-4.

3. Al-Omran M. True ulnar artery aneurysm of the hand in an 18-month-old boy: A case report. J Vasc Surg 2007;45:841-3.

4. Sarkar R, Arnold G, Coran AG, et al. Arterial aneurysms in children: Clinicopathologic classification. J Vasc Surg 1991;13:47-57.

5. Nguyen D, Murison M. Ulnar artery aneurysm in a patient with Marfan's syndrome. J Plast Reconstr Aesthet Surg 2006:59:1131-2.

6. San Vicente B, Castanon M, Mulet J, et al. Aneurisma postraumatico de la arteria humeral. Cir Pediatr 2000;13:84-6.

7. Venkatanarasimha N, Manghat NE, Wells IP. Unusual presentation of ulnar artery aneurysm and dissection with associated anomalous radial artery: Appearances on multi-detector row CT angiography. Emerg Radiol 2007;14:101-4.

8. Offer GJ, Sulley L. Congenital aneurysm of the ulnar artery in the palm. J Hand Surg (Br) 1999;24:735-7.

9. Witt PD, Bowen KA, Johansen K. True ulnar artery aneurysm of the hand in an 8-year-old boy. Plast Reconstr Surg 2003;111:2475-6.

10. Deune EG, McCarthy EF. Reconstruction of a true ulnar artery aneurysm in a 4-year-old patient with radial artery agenesis. Orthopedics 2005;28:1459-61.

11. Rothkopf DM, Bryan DJ, Cuadros CL, et al. Surgical management of ulnar artery aneurysms. J Hand Surg (Am) 1990;15:891-7. 